\title{
Discrete Element Analysis of Friction Coefficient on Granular Accu- mulation
}

\author{
Qinliang $\mathrm{Li}^{1, \mathrm{a}}$, Bin Zhao ${ }^{2, \mathrm{~b}}$, Bo Wang ${ }^{1}$, Bangchun Wen ${ }^{1}$ \\ ${ }^{1}$ School of Mechanical Engineering and Automation, Northeastern University, Shenyang, China \\ ${ }^{2}$ School of Mechanical \& Electrical Engineering, Henan University of Technology, Zhengzhou, \\ China \\ aliqinliang@vip.qq.com, 'bhaobin978@163.com
}

Keywords: friction coefficient, granular accumulation, angle of repose, DEM

\begin{abstract}
Discrete element method (DEM) is applied to study the granular accumulation problem. Using Herz-Mindlin (no slip) model to simulate particles and container model is also established by software. When the container elevates, the process of granular falling and collision can be observed. Detailed analysis of that the impact of static and rolling friction coefficient for particles - particles, particles - flat on angle of repose is accomplished. The variation law is also further validated from the energy point of view. The results show that rolling friction has a greater impact on angle of repose than static friction, and rolling friction coefficient among particles play the more prominent role in the two kinds of rolling friction. The research method and results provide a theoretical reference for the granular movement and DEM analysis.
\end{abstract}

\section{Introduction}

Friction inevitably accompanies the granular movement, whose strength is limited. Angle of repose is the angle between the natural slope and horizon when the static granular accumulation process is completed under some assigned conditions.

Angle of repose reflects the macroscopic granular characteristics, which is related with machinery, transportation, metallurgy, energy and other practical applications of engineering and agriculture. For instance, angle of repose affects the upgrading angle of conveyor in the mine production system, which results in large arrangement area, big project and long structure. All above have severe impacts on research and development of bulk material handling and stacker equipments.

As granular geometries and movement patterns are very complex, it is difficult to describe bulk material by the continuous function. In recent years, some scholars individually conducted detailed studies on the granular accumulation characteristic by molecular dynamics and discrete element method in order to calculate and predict the granular movement state more accurately [1][2],[3].

\section{Research Method}

Discrete element method (DEM) is based on Newton's second law. First, particles are divided into collections of discrete granular cell, the movement equation of each cell is established by associating constitutive relations between particles, then different relationships between force and displacement can be solved by dynamic relaxation method. Thus the overall granular movement state is obtained.

With the rapid development of computer technology in recent years, three-dimensional simulation software such as PFC2D/3D, EDEM has become the powerful tool for analyzing the granular behavior. Discrete element simulation can obtain datas which the experiment can't easily measure. And the post-processing tools of DEM software allows us investigate and understand the situation 
of model through data analysis, visualization tools and others, which make us better solve practical problems. This paper aims to reveal basic characteristics and laws of mechanical properties using discrete element method, providing a basis for the selection of friction coefficient in future simulation work.

\section{Container modeling}

Granular accumulation may seem simple, but its internal mechanism is quite complex. Different devices have different heap-arch effect; therefore, simulation conditions should be selected according to their similar accumulation methods.

In this paper, the research is based on the injection method. The circular funnel and bottom flat is established in software which doesn't have thickness, simultaneously ensuring that the diameter of flat is larger than the bottom diameter of generated heap. Circular funnel bottom opening diameter: $50 \mathrm{~mm}$, top opening diameter: $160 \mathrm{~mm}$, height: $125 \mathrm{~mm}$. The opening diameter should be at least $5 \sim 6$ times to the granular diameter, because the congestion may be caused by force arch when the generated granular size is large.
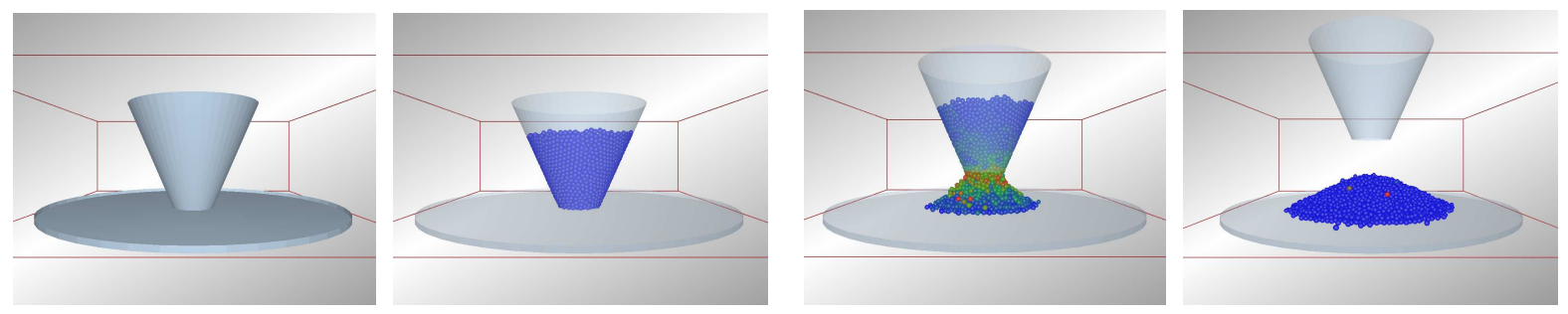

Fig. 1. Granular accumulation process

Table 1 Material parameters

\begin{tabular}{ccc}
\hline Parameters & Particle & Flat \\
\hline Density[kg/m $\left.\mathrm{m}^{3}\right]$ & 1600 & 1500 \\
Poisson's ratio & 0.28 & 0.47 \\
Shear modulus[Gpa] & 1.98 & 0.00267 \\
Restitution coefficient & p-p 0.5 & p-f 0.45 \\
\hline
\end{tabular}

In the simulation, the bottom funnel opening keeps contact with flat at first. Then the funnel upgrades at vertical speed $0.05 \mathrm{~m} / \mathrm{s}$ after the stability of generated particles. This can make the vertical distance between the falling particles and heap top relatively constant on one hand, avoiding excessive impacts on the heap, the upgrade also help to enhance the falling of internal particles in funnel on the other hand. Particles gradually fall from funnel and accumulate on the flat. The whole simulation doesn't complete until the entire stable granular accumulation forms on the flat. Specific process is shown in Figure 1, and the material parameters for the simulation are in Table 1.

\section{Angle of repose measurement}

The surface shape of heap generated by injection method is similar to cone. The outline curve can be divided into three parts: heap top, slope, heap tail. Due to the continuous impact of falling particles, the curve of heap top is arc. The curve of heap tail is nearly the same as heap top, but in the opposite direction[5]. The angle measurement is mainly in the slope area. In the traditional method, the direct measurement is on the relatively regular surface using angle tools; another is painting the angle plane on the basis of measuring the height and bottom diameter of cone and calculating its angle value. Due to internal friction of the heap, the accumulation surface often shows convex and concave or other irregular shapes, which have severe effect on the accuracy of angle measurement[6]. 
In the simulation, Using the post-processing tools of DEM software we can establish a grid area in the generated heap, and then export center coordinate of each particle in the area, as shown in Figure 2. Using Matlab to analyze its geometrical information further: Dividing the heap along the $\mathrm{x}$-axis into vertical equal width intervals, searching particles in each interval with largest $y$ value, and obtaining the surface information, then proceeding linear curve fitting to the approximation surface using least square method. The fitting range does not include heap top and heap tail,

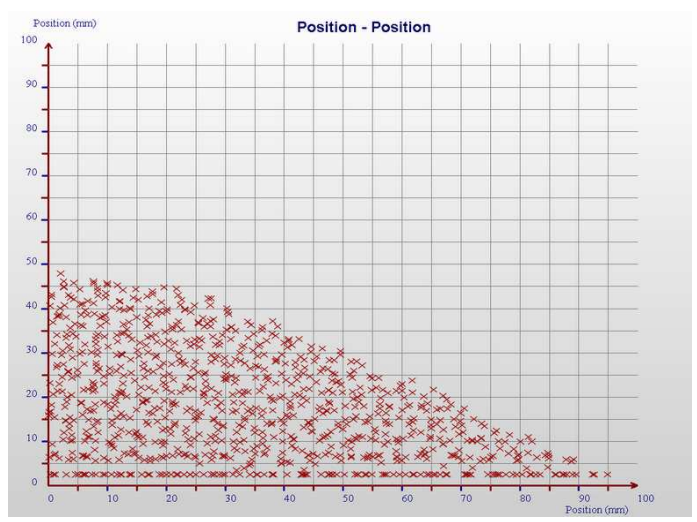

Fig. 2. Core position of particles because these two regions are both arc. Finally we can obtain angle of repose by applying arc tangent to the fitting line.

\section{Friction coefficient impact}

When particle get in contact with the heap, it need a sliding and rolling process to consume initial energy and achieve steady state at last. In the process the sliding friction and rolling friction plays a suppression role in granular movement and rotation, largely determining the stability of contacts.

The granular surface roughness directly affects macroscopic accumulation characteristic of the heap. It is difficult to fully capture the impact brought by the variation of friction coefficient in experiments. When the contact area between particles is small, the selflocking caused by the granular surface shape and adhesion also has significant effect on angle of repose in addition to friction coefficient. In order to simplify the accumulation problem, The paper only uses the Herz-Mindlin model[7] in research which is applicable for dry granular ball.

In this model, when two particles collide, the collision force on contact point can be decomposed into two kinds of normal and tangential force. The extreme value of tangential force equals the product of static friction coefficient and normal force. When tangential force is larger than ex-

Table 2 Simulation parameters

\begin{tabular}{cc}
\hline \multicolumn{2}{c}{ Granular parameters } \\
\hline $\begin{array}{c}\text { Diameter }[\mathrm{mm}] \\
\text { Granular number }\end{array}$ & 5000 \\
\hline \multicolumn{2}{c}{ Friction coefficients } \\
\hline p-p static $\mu_{\mathrm{s}, \mathrm{pp}}$ & $0.2,0.4,0.6,0.8$ \\
$\mathrm{p}-\mathrm{f}$ static $\mu_{\mathrm{s}, \mathrm{pf}}$ & $0.2,0.4,0.6,0.8$ \\
$\mathrm{p}-\mathrm{p}$ rolling $\mu_{\mathrm{r}, \mathrm{pp}}$ & $0.05,0.1,0.2,0.3$ \\
$\mathrm{p}-\mathrm{f}$ rolling $\mu_{\mathrm{r}, \mathrm{pf}}$ & $0.05,0.1,0.2,0.3$ \\
\hline
\end{tabular}

treme value, the sliding will ocurr. At this moment, the value of sliding friction is substituted by the maximum static friction, and the tangential elastic deformation between particles also continually hinder the sliding. Rolling friction coefficient determines the rolling friction force and torque, playing a important role in inhibiting rolling.

To better understand the impact of friction coefficient on angle of repose, the simulation are carried on for different static and rolling friction coefficient of particle - particle and particle - flat, the selected simulation parameters are in Table 2. 
$\mu_{\mathrm{s}, \mathrm{pp}}$ and $\mu_{\mathrm{r}, \mathrm{pp}}$ are both important factors which influence angle of repose. In order to study their effects on angle, 16 groups of simulation are individually carried on according to the parameters in table 2 . At this point $\mu_{\mathrm{s}, \mathrm{pw}}$ and $\mu_{\mathrm{r} \text {,pw }}$ is respectively as 0.2 and 0.05 , then using the results of these simulations to compile statistics, drawing out the angle variation graph, as shown in Figure 3. Granular accumulation forms are shown in Figure 4 with $\mu_{\mathrm{s}, \mathrm{pp}} 0.1$ and $\mu_{\mathrm{r}, \mathrm{pp}} 0.2,0.4,0.6,0.8$ respectively. As the above graphs show,
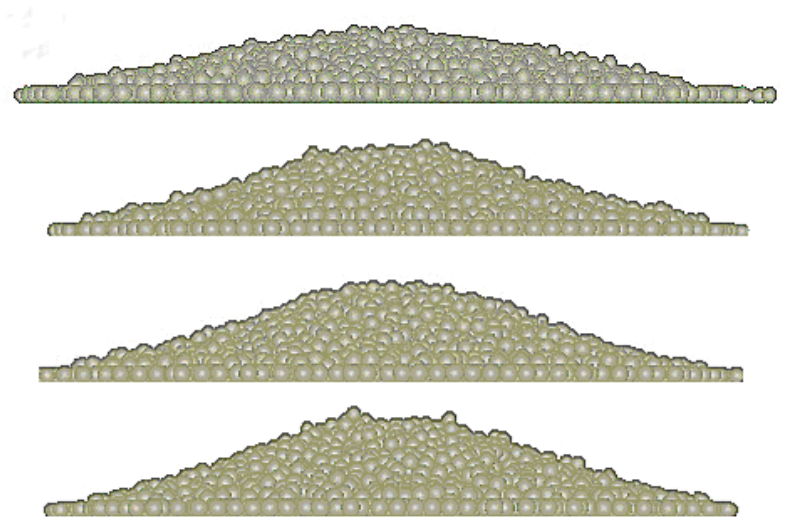

Fig. 4. Granular formation as a function of $\mu_{\mathrm{r}, \mathrm{pp}}=0.1, \mu_{\mathrm{s}, \mathrm{pp}}=0.2,0.4,0.6,0.8$
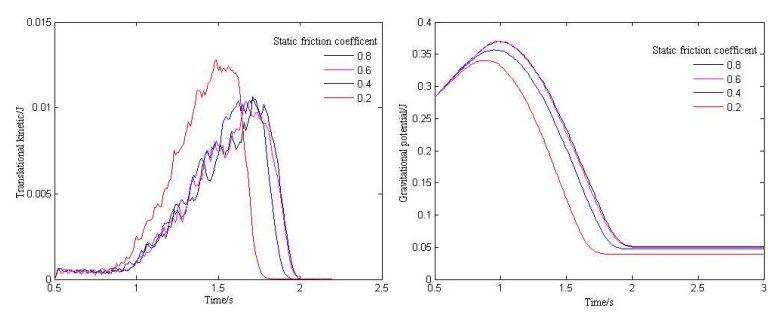

(a) Translational kinetic (b) Gravitational potential

Fig. 5. Granular energy varying with $\mu_{\mathrm{r}, \mathrm{pp}}=0.1$, $\mu_{\mathrm{s}, \mathrm{pp}}=0.2,0.4,0.6,0.8$

Funnel begins to upgrade from $0.5 \mathrm{~s}$, the larger $\mu_{\mathrm{s}, \mathrm{pp}}$ is , the more slowly internal particles discharges. The lifting of funnel results in that more remain particles upgrade with funnel, so the peak of overall translational kinetic is smaller, but the peak of total gravitational potential is greater.

After a period of accumulation, an increasing number of particles emitted, The total translational kinetic is close to zero, while the total gravitational potential is decreasing and approaching a stable value after the heap is constant. The lager $\mu_{\mathrm{s}, \mathrm{pp}}$ is, the more slowly the velocity

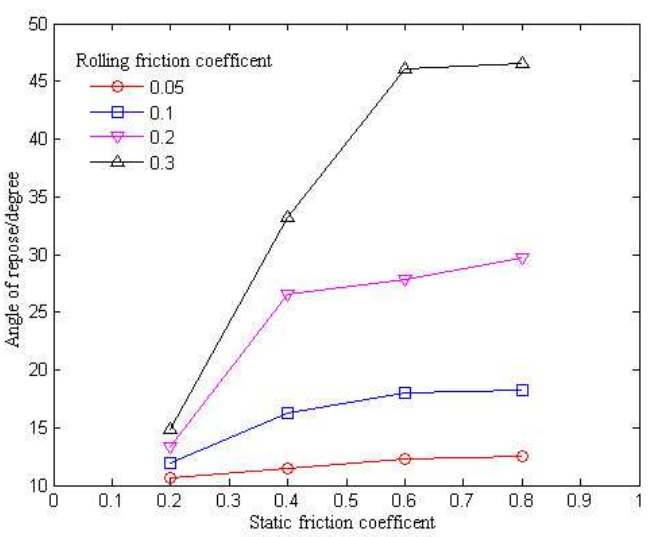

Fig. 3. Angle of repose varying with friction coefficient between particles

When $\mu_{\mathrm{s}, \mathrm{pp}}$ is small, particles drop from the funnel and easily spread around so the heap is difficult to form on flat. Most of particles spread on the bottom which also increases the bottom diameter of heap. For the above reason the angle becomes small. As $\mu_{\mathrm{s}, \mathrm{pp}}$ gradually increases, particles are difficult to slide between the contacts, enlarging the supporting role, thus the angle increases at last. The angle changes obviously only when $\mu_{\mathrm{s}, \mathrm{pp}}$ increases from 0.2 to 0.4 in graph, but the increased rate is not great in other cases, so it can be seen that the role of static friction between particles is limited. Figure 5 compares the real-time graph of translational kinetic and gravitational potential in four groups of conditions.
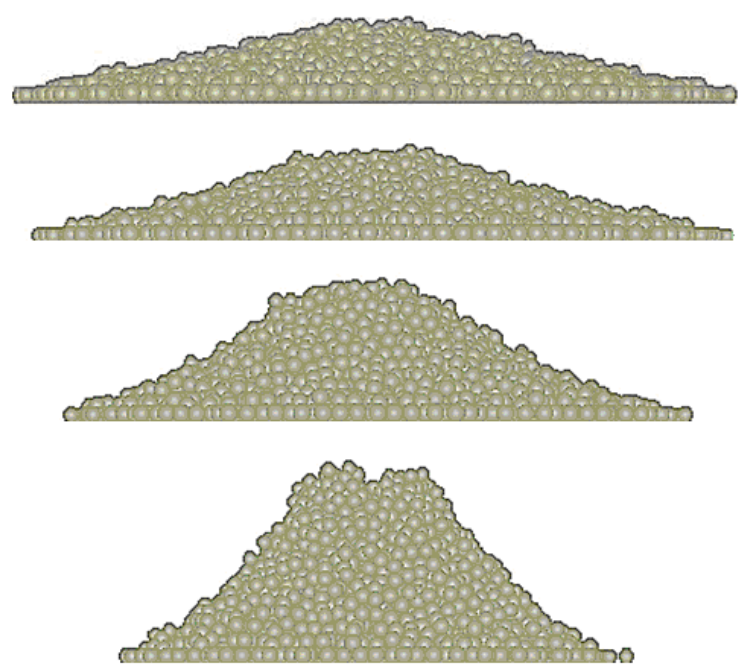

Fig. 6. Granular formation varying with

$$
\mu_{\mathrm{s}, \mathrm{pp}}=0.6, \mu_{\mathrm{r}, \mathrm{pp}}=0.05,0.1,0.2,0.3
$$




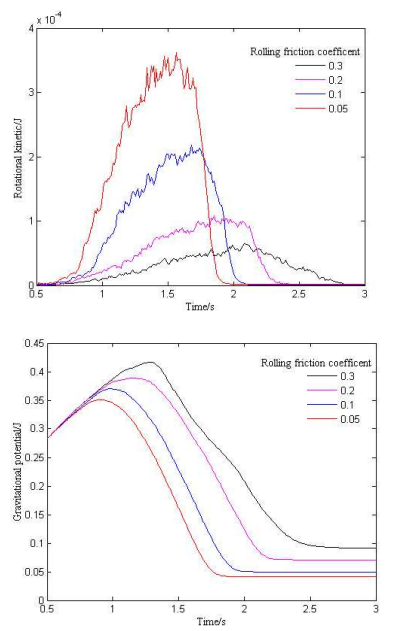

(a) Rotational kinetic (b) Gravitational potential Fig. 7. Granular energy varying with $\mu_{\mathrm{s}, \mathrm{pp}}=0.6, \mu_{\mathrm{r}, \mathrm{pp}}=0.05,0.1,0.2,0.3$

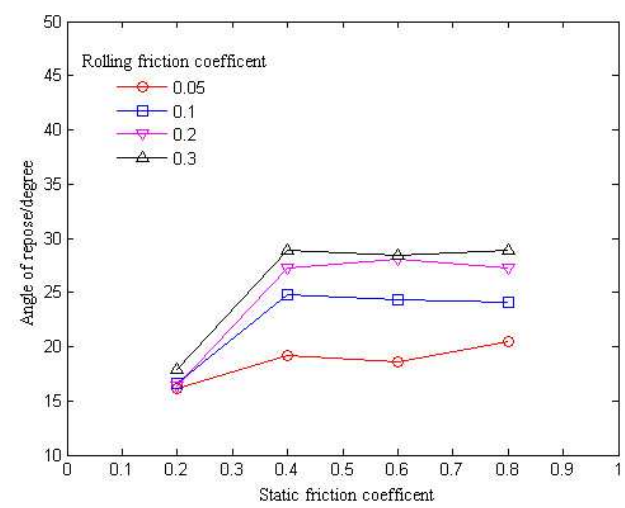

Fig. 8. Angle of repose varving with fric-
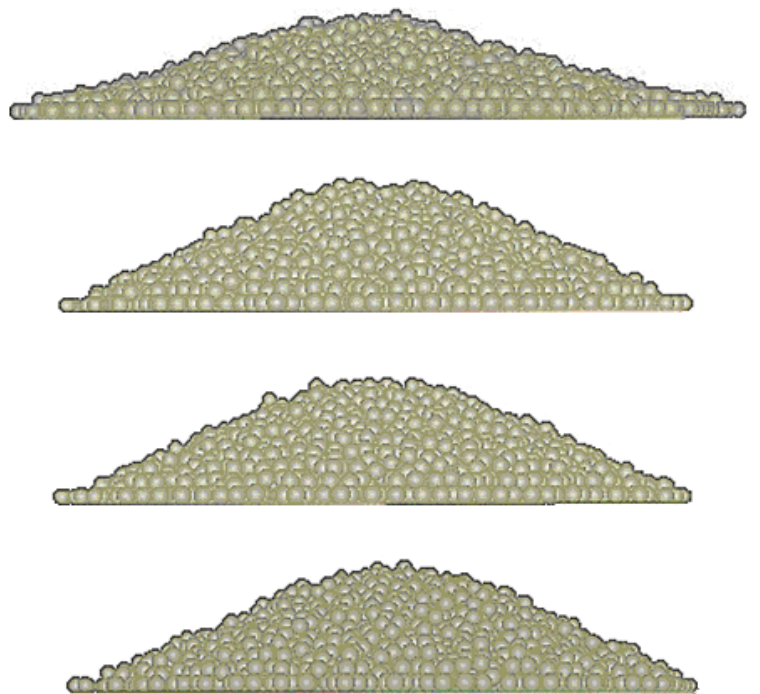

Fig. 9. Granular formation varying with $\mu_{\mathrm{r}, \mathrm{pf}}$ $=0.1, \mu_{\mathrm{s}, \mathrm{pf}}=0.2,0.4,0.6,0.8$

but angle changes little with $\mu_{\mathrm{s}, \mathrm{pf}} 0.4,0.6,0.8$ respectively. This is mainly because static friction between particles and flat is enough to limit their relative sliding and promote ac cumulation more easily when the friction coefficient is greater than 0.4. Therefore, at this time the angle is most determined by $\mu_{\mathrm{r}, \mathrm{pp}}$. of translational kinetic energy tends to zero, and the constant value which gravitational potential approachs is larger, illustrating that the overall height of heap is higher. All the above verify the impact of $\mu_{\mathrm{s}, \mathrm{pp}}$ on angle of repose from the energy point of view.

The increase of $\mu_{\mathrm{r}, \mathrm{pp}}$ inhibits the relative rolling and also makes angle of repose increase at the same time. As the main granular movement is rolling and most of them are between particles, comparatively $\mu_{\mathrm{r}, \mathrm{pp}}$ influences angle of repose more apparently. As is discovery in simulation: with $\mu_{\mathrm{r}, \mathrm{pp}}$ enlargement, the heap presents apparent convex, concave and other irregular shape when the static friction between particles has been fully limited, as shown in Figure 6. In Figure 7, the variation trend of rotational kinetic is more obvious than translational kinetic energy when the effect of $\mu_{\mathrm{s}, \mathrm{pp}}$ is studied. The constant value which the gravitational potential approachs apparently increases when the heap stablizes. The fluctuation of heap shape is most obvious when $\mu_{\mathrm{r}, \mathrm{pp}}$ is 0.3 . Thus, $\mu_{\mathrm{r}, \mathrm{pp}}$ has a more significant impact on angle.

As is similar with the friction coefficient between particles, the contacts between particles and bottom flat also have impact on angle. Here, 16 groups of simulation are also accomplished to analyze the variation effect of $\mu_{\mathrm{r}, \mathrm{pf}}$ and $\mu_{\mathrm{s}, \mathrm{p} f}$, at this point $\mu_{\mathrm{s}, \mathrm{pp}}$ and $\mu_{\mathrm{r}, \mathrm{pp}}$ is individually set as 0.4 and 0.1 . Figure 8 is the variation of angle. As is shown in Figure 9 and 10, angle of repose has significant variation only when $\mu_{\mathrm{s}, \mathrm{pf}}$ changes from 0.2 to 0.4 ,
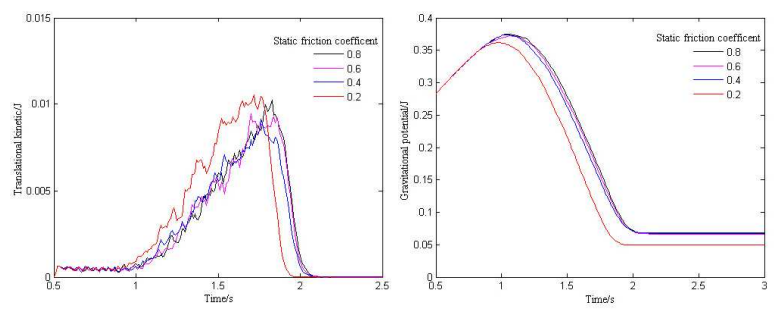

(a) Translational kinetic (b) Gravitational potential

Fig. 10. Granular energy varying with $\mu_{\mathrm{r}, \mathrm{pf}}=$ $0.1, \mu_{\mathrm{s}, \mathrm{pf}}=0.2,0.4,0.6,0.8$ 
In contrast, the influence of $\mu_{\mathrm{r}, \mathrm{pf}}$ is also sm- aller than $\mu_{\mathrm{r}, \mathrm{pp}}$. As is shown in figure 11, 12 with
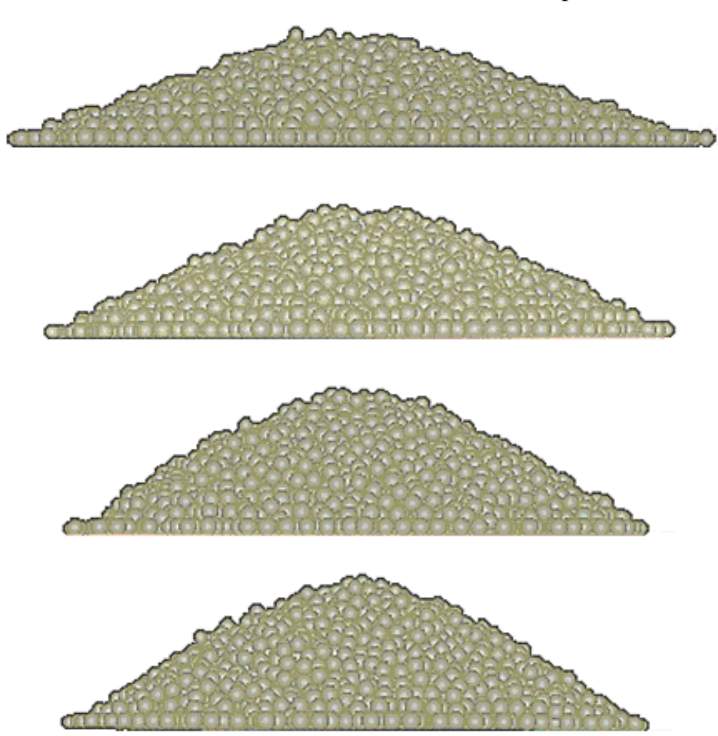

Fig. 11. Granular formation as a function of $\mu_{\mathrm{s}, \mathrm{pf}}=0.6, \mu_{\mathrm{r}, \mathrm{pf}}=0.05,0.1,0.2,0.3$
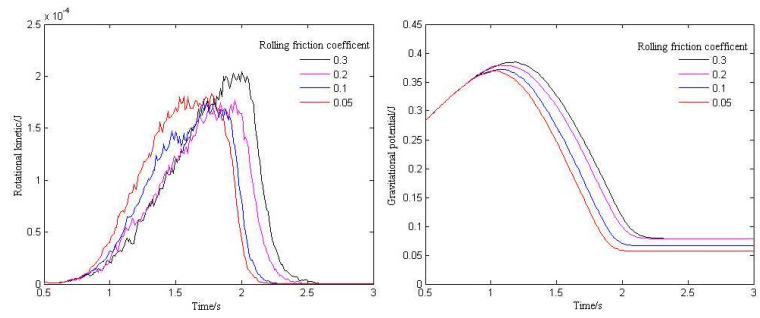

(a) Rotational kinetic (b) Gravitational potential Fig. 12. Granular energy varying with $\mu_{\mathrm{s}, \mathrm{pf}}=$ $0.6, \mu_{\mathrm{r}, \mathrm{pf}}=0.05,0.1,0.2,0.3$ $\mu_{\mathrm{s}, \mathrm{pf}}=0.6, \mu_{\mathrm{r}, \mathrm{pf}}=0.05,0.1,0.2,0.3$ respectively, angle of repose gradually increases. The heap outline changes from initial approximately linear shape into obvious convex, and the bottom diameter is gradually reduced. This is mainly due to that the weakening of rolling effect at the bottom inhibits the particles to spread around, consequently offering better supporting for the subsequent falling particles.

\section{Conclusion}

After comparing these four kinds of friction coefficient, we find that in the accumulation process the influence of rolling friction is bigger than static friction and in rolling friction the friction among particles plays the more prominent role, which can significantly affect the angle and change the heap shape. Rolling friction betweem particles and flat only limits the rolling of bottom particle which can affect the geometry of heap tail and provide strong supporting for the top particles. When the static friction of particles - particles and particles - flat reaches a certain value, their influence become not obvious. This is because that the mutual sliding has been fully suppressed, and rolling friction plays a more important role.

\section{References}

[1] Y. Grasselli, H.J. Hermann. On the angles of dry granular heaps. Physica A, 1997

[2] Y.C. Zhou, B.H. Xu, A.B. Yu, P. Zulli. An experimental and numerical study of the angle of repose of coarse spheres. Powder Technology, 2002

[3] Li Yanjie, $\mathrm{Xu}$ yong, C. Thornton. A comparison of discrete element simulations and experiments for 'sandpiles' composed of spherical particles. Powder Technology, 2005

[4] Wang Yong Jia, Xing Jibo. Discrete element method and its application in rock mechanics. Liaoning: Northeast University Press, 1991

[5] Y. Grasselli, H.J. Herrmann. Shapes of heaps and in silos. European Physical Journal B, 1999

[6] Zlobecki, J. Frczek, J. Zemanek. Assessment of angle of repose of granular plant material using computer image analysis. Journal of Food Engineering, 2007

[7] DEM Solutions. EDEM2.2.1 programming guide, 2010 